Japan. J. Math.

Vol. 16, No. 2, 1990

\title{
An application of the second microlocalization at the boundary to the extension of solutions of differential systems
}

\author{
By Giuseppe ZamPIERI
}

(Received May 26, 1989)

§ 0. We use the theory of microlocalization of sheaves of [K-S2], and especially its formulation in [S] for boundary value problems, to treat the extension of regular solutions of systems of P.D.E. across 1-codimensional singular sets. Let $M$ be a real analytic manifold, $X$ a complexification of $M, N$ an analytic hypersurface of $M, Y$ a complexification of $N, \Omega$ an open component of $M \backslash N$ at $x \in N$. For a suitable involutive manifold $V \subset \dot{T}_{M}^{*}$, invariant under the Hamiltonian flow of $N \times_{M} \dot{T}_{M x}^{*}$, we introduce a new complex $\mathscr{B}_{\Omega_{\mid X}}^{a}$ of hyperfunctions in $\Omega$ with real analytic parameters (of also [Ô2]) and study its applications to non-characteristic boundary value problems. In particular we show that the trace morphism preserves the analytic parameters. The analysis of $\mathscr{B}_{\Omega_{\mid X}}^{a}$ could be performed from the viewpoint of the 2nd microlocalization at the boundary along $V$ developped in [U-Z]; but we do not need to refer to such a general theory for the purpose of the present paper. We then consider a differential system $\mathscr{M}$ at $x, x \in N$, for which $Y$ is non-characteristic. We denote by $\tilde{V}$ the union of the leaves of $V^{c}$ issued from $V$, and by $\rho$ the projection $Y \times_{X} T^{*} X \rightarrow T^{*} Y$. We take a closed set $S \subset N$ with $x \in \partial S$ and make the following hypotheses: $\mathscr{M}$ is microhyperbolic relative to $\tilde{V}$ to any $\theta \in \dot{T}_{N}^{*} M_{x}$; char $\mathscr{M} \cap \rho^{-1} \rho\left(\{x\} \times_{M} V\right) \subset V$; $i N_{X}^{*}(S) \subset \rho\left(\{x\} \times_{M} V\right)$. We then prove that $H^{0}\left(\mathscr{B}_{M \mid X}^{a}\right)$-solutions of $\mathscr{M}$ on $M \backslash S$ extend to $M$ at $x$. (Here $H^{0}\left(\mathscr{B}_{M \mid X}^{a}\right)$ is the sheaf of hyperfunctions depending real analytically on the variables transversal to the leaves of $V$.) Under some additional hypotheses on "propagation in the interior", we also obtain the extension of $\mathscr{A}_{M}$-solutions. The basic method of the paper, namely the estimation of microsupport for traces of solutions to single operators (resp. to systems), is due to Kaneko [Kan 1] (resp. Oaku [Ô 1]).

\section{§1. The complexes $\mathscr{B}_{Q \mid X}^{2}$ and $\mathscr{B}_{a \mid X}^{a}$}

Let $M=M^{\prime} \times L$ be real analytic manifolds with complexifications $X=$ $X^{\prime} \times Z$ and dimensions $n=n_{1}+n_{2}$. For a locally closed set $A=A^{\prime} \times L$ of $M$, put $\tilde{A}=A^{\prime} \times Z$ and define (cf. [K-S-2], [S]) 


$$
\begin{gathered}
\mathscr{C}_{A \mid X}^{h}=\mu_{\tilde{A}}\left(\mathcal{O}_{X}\right) \otimes \omega_{M^{\prime} / X^{\prime}}\left[n_{1}\right], \\
\mathscr{B}_{A \mid X}^{2}=R \Gamma_{T^{*} X^{\prime} \times L}\left(\mathscr{C}_{A \mid X}^{h}\right) \otimes \omega_{L / Z}\left[n_{2}\right] .
\end{gathered}
$$

We often consider the case $A=M$ or $A=N$ for an analytic submanifold $N=$ $N^{\prime} \times L$ of $M$ of codimension 1 , or else $A=\Omega$ where $\Omega=\Omega^{ \pm}$are the components of $M \backslash N$. The following triangle will play an essential role:

$$
\mathscr{B}_{N \mid X}^{2} \longrightarrow \mathscr{B}_{M \mid X}^{2} \longrightarrow \mathscr{B}_{\Omega+\mid X}^{2} \oplus \mathscr{B}_{\Omega-\mid X}^{2} \longrightarrow{ }^{+1} \text {. }
$$

REMARK 1.1. By the results of [U-Z] we colud give a canonical definition of the complexes $\mathscr{C}_{* \mid X}^{2}$ and $\mathscr{B}_{* \mid X}^{2}, *=M, N, \Omega$, associated to a smooth conic regular involutive manifold $V \subset \dot{T}_{M}^{*} X$ such that

$$
\begin{aligned}
& V \text { and } N \times_{M} \dot{T}_{M}^{*} X \text { intersect transversally, } \\
& \text { and } N \times_{M} V \text { is regular involutive. }
\end{aligned}
$$

We recall from [K-L] that for $*=M, N, \mathscr{B}_{* \mid X}^{2}$ is concentrated in degree 0

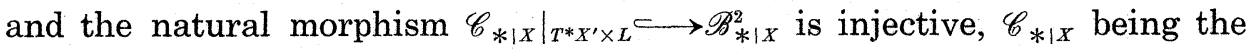
sheaf of usual microfunctions. (As for the case $*=\Omega$ it is proven in [U-Z] that $\left(\mathscr{B}_{Q_{\mid X} X}^{2}\right)_{T_{M}^{*}, X^{\prime} \times L}$ is concentrated in degree 0 but that the corresponding result on injectivity does not hold any more. However this is not needed here.)

We set now:

$$
\mathscr{B}_{\Omega \mid X}^{a}=R \Gamma_{M}\left(\left(\mathcal{O}_{X}\right)_{D^{\prime} \times Z}\right) \otimes \omega_{L / Z}\left[n_{2}\right] .
$$

We have a distinguished triangle

$$
\mathscr{B}_{\Omega \mid X}^{a} \longrightarrow R \Gamma_{\Omega}\left(\mathscr{B}_{M}\right) \longrightarrow R \dot{\pi}_{*}\left(\mathscr{B}_{\Omega \mid X}^{2}\right) \longrightarrow{ }^{+1},
$$

$\mathscr{B}_{M}$ being the sheaf of hyperfunctions. (We remark that (1.6) is obtained by applying $R \Gamma_{M}(\cdot) \otimes \omega_{M / X}[n]$ to the triangle

$$
\left(\mathcal{O}_{X}\right)_{\bar{\Omega}^{\prime} \times Z} \otimes \omega_{M^{\prime} / X}\left[-n_{1}\right] \longrightarrow R \Gamma_{\Omega^{\prime} \times Z}\left(\mathcal{O}_{X}\right) \longrightarrow R \dot{\pi}_{*} \mu_{\Omega^{\prime} \times Z}\left(\mathcal{O}_{X}\right) \longrightarrow{ }^{+1} .
$$

We similarly define

$$
\begin{aligned}
& \mathscr{B}_{M \mid X}^{a}=R \Gamma_{M}\left(\left(\mathcal{O}_{X}\right)_{M^{\prime} \times Z}\right) \otimes \omega_{L / Z}\left[n_{2}\right], \\
&\text { (resp. } \left.\quad \mathscr{B}_{N \mid X}^{a}=R \Gamma_{M}\left(\left(\mathcal{O}_{X}\right)_{N^{\prime} \times Z}\right) \otimes \omega_{L / Z}\left[n_{2}\right]\right),
\end{aligned}
$$

and get (1.6) with $\Omega$ replaced by $M$ (resp. $N$ and with the first term shifted by -1). One also proves that $\mathscr{B}_{* \mid X}^{a}, *=M, N$ or $\Omega$, are concentrated in degrees 0,1 with $H^{1}\left(\mathscr{B}_{* \mid X}^{a}\right) \neq 0$. For $*=M$ this follows from the analogous of (1.6), from the flabbiness of $\mathscr{B}_{M \mid X}^{2}$ and the non-surjectivity of $\left.\mathscr{C}_{M}\right|_{T_{M}^{*}, X^{\prime} \times L} \longrightarrow$ $\mathscr{B}_{M \mid X}^{2}$. As for $*=N$ the degree is $\leqq 1$ (by the flabby dimension 1 of $\mathscr{B}_{N \mid X}^{2}$ ) and 
this is in fact 0,1 (by the edge of the wedge theorem for $\left.\left(\mathcal{O}_{X}\right)_{N^{\prime} \times Z}\right)$. Moreover $H^{1}\left(\mathscr{B}_{N \mid X}^{2}\right) \neq 0$ by an argument of the type of the dummy variable (i.e. by microlocalization of the exact sequence: $0 \rightarrow \mathcal{O}_{X} \rightarrow \mathcal{O}_{X} \rightarrow j_{*} \mathcal{O}_{Y} \rightarrow 0$, $Y$ being a complexification of $N$ and $j: Y \hookrightarrow X$ the embedding) and by the corresponding result for $\mathscr{B}_{M \mid X}^{2}$. Finally the case $*=\Omega$ obviously follows from those $*=M$ and $*=N$. The detailed study of the complex (1.5) is left to [U-Z]; we only treat here its applications to boundary value problems. Thus let $\mathscr{M}$ be a coherent $\mathscr{D}_{X}$-module on an open set of $M$. We assume all through this section that $Y$, the complexification of $N$, is non-characteristic for $\mathscr{M}$.

Proposition 1.2. The natural morphisms

$$
\left.H^{0}\left(R \operatorname{Hom}_{\mathscr{P}_{X}}\left(\mathscr{M}, \mathscr{C}_{\Omega_{\mid X} X}\right)\right)\right|_{T^{*} X^{\prime} \times L} \longrightarrow H^{0}\left(R \operatorname{Hom}_{\mathscr{P}_{X}}\left(\mathscr{M}, \mathscr{B}_{\Omega^{\prime} X}^{2}\right)\right),
$$

and

$$
H^{0}\left(R \operatorname{Hom}_{\mathscr{D}_{X}}\left(\mathscr{M}, \mathscr{B}_{\Omega \mid X}^{a}\right)\right) \longrightarrow \operatorname{Hom}_{\mathscr{O}_{X}}\left(\mathscr{M}_{,} \Gamma_{\Omega}\left(\mathscr{B}_{M}\right)\right),
$$

are injective. (cf. [S-Z 1] as for (1.7))

Proof. By the results of [K-L] it is enough to prove (1.7) and (1.8) in $T_{N^{\prime}}^{*} X^{\prime} \times L$ and $N$ respectively. As for (1.7) let us consider the commutative diagram

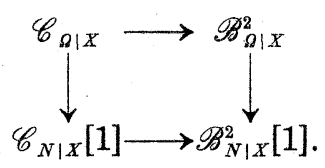

We observe that

$$
H^{0}\left(R \operatorname{Hom}_{\mathscr{I}_{X}}\left(\mathscr{M}, \mathscr{C}_{\Omega_{\mid X}}\right)\right) \longrightarrow H^{1}\left(R \operatorname{Hom}_{\mathscr{P}_{X}}\left(\mathscr{M}, \mathscr{C}_{N \mid X}\right)\right)
$$

is injective, due to the triangle: $\mathscr{C}_{N \mid X} \rightarrow \mathscr{C}_{\bar{\Omega} \mid X} \rightarrow \mathscr{C}_{\Omega \mid X} \rightarrow^{+1}$ and to:

$$
\operatorname{Hom}_{\mathscr{D}_{X}}\left(\mathscr{M}, \mathscr{C}_{\bar{\Omega}_{\mid X}}\right)_{\pi^{-1}(N)}=0 .
$$

(The latter vanishing follows in turn from the unique continuation property for $\mathscr{C}_{\left.\tilde{g}_{\mid X}\right|_{t^{-1}(N)}}$ due to Kataoka [Kat] together with the non-characteristicity of $Y$.) We also have

$$
\left.\operatorname{Hom}_{\mathscr{O}_{X}}\left(\mathscr{M}, \mathscr{B}_{N \mid X}^{2} / \mathscr{C}_{N \mid X}\right)\right|_{T_{N}^{*} X^{\prime} \times L}=0,
$$

by division formulas for $\mathscr{C}_{N \mid X}$ and $\mathscr{B}_{N \mid X}^{2}$ (cf. [S-Z 2]). The injectivity of (1.7) follows at once.

As for the injectivity of (1.8), this is equivalent, in view of (1.6) to:

$$
H^{-1}\left(R \operatorname{Hom}_{\mathscr{P}_{X}}\left(\mathscr{M}, R \dot{\pi}_{*}\left(\mathscr{B}_{\Omega \mid X}^{2}\right)\right)\right)=0 .
$$


On the other hand this follows from: $\operatorname{Hom}_{\mathscr{O}_{X}}\left(\mathscr{M}, \mathscr{B}_{N \mid X}^{2}\right)=0$ which is still a consequence of division formula for $\mathscr{B}_{N \mid X}^{2}$.

Let $\mathscr{M}_{Y}$ denote the induced system of $\mathscr{M}$ on $Y$, and let $\gamma: \operatorname{Hom}_{\mathscr{O}_{X}}(\mathscr{M}$, $\left.\Gamma_{\Omega}\left(\mathscr{B}_{M}\right)\right)\left.\right|_{N} \rightarrow \operatorname{Hom}_{\mathscr{O}_{Y}}\left(\mathscr{M}_{Y}, \mathscr{B}_{N}\right)$ be the trace morphism (cf. [S]) By collecting all above results we get

Proposition 1.3. We have

$$
\begin{aligned}
& H^{0}\left(R \operatorname{Hom}_{\mathscr{O}_{X}}\left(\mathscr{M}, \mathscr{B}_{\Omega \mid X}^{a}\right)\right)_{x} \\
& \quad=\left\{u \in \operatorname{Hom}_{\mathscr{O}_{X}}\left(\mathscr{M}, \Gamma_{\Omega}\left(\mathscr{B}_{M}\right)\right)_{x}: S S(\gamma(u)) \cap\left(\dot{T}_{N^{\prime}}^{*} Y^{\prime} \times L\right)=\varnothing\right\}, \quad x \in N .
\end{aligned}
$$

Proof. Let $\mathscr{F}=R \operatorname{Hom}_{\mathscr{O}_{X}}\left(\mathscr{M}, \mathcal{O}_{X}\right)$, put $\widetilde{\Omega}=\Omega^{\prime} \times Z$, and note that the natural diagram

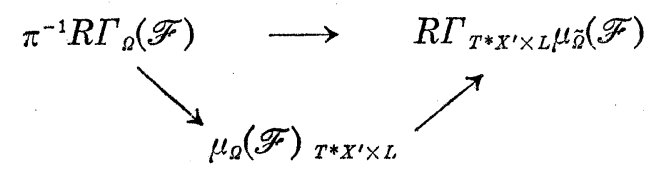

is commuting. Thus recalling (1.6) and applying Proposition 1.2, we get

$$
\begin{aligned}
& H^{0}\left(R \operatorname{Hom}_{\mathscr{O}_{X}}\left(\mathscr{M}, \mathscr{B}_{\Omega_{\mid X}}^{a}\right)\right)_{x} \\
& \quad=\left\{u \in \operatorname{Hom}_{\mathscr{O}_{X}}\left(\mathscr{M}, \Gamma_{\Omega}\left(\mathscr{B}_{M}\right)\right)_{x}: S S_{\Omega}^{\mu, 0}(u) \cap\left(\dot{T}^{*} X^{\prime} \times L\right)=\varnothing\right\},
\end{aligned}
$$

where $S S_{\Omega}^{\mathscr{A}, 0}(u)$ is the support of $u$ identified to a section of $H^{0}\left(R \operatorname{Hom}_{\mathscr{P}_{X}}(\mathscr{M}\right.$, $\left.\mathscr{C}_{Q_{\mid X}}\right)$ ) (cf. [S]). According to [S] this is in turn equivalent to (1.11).

REMARK 1.4. When considering $\mathscr{B}_{M \mid X}^{a}$ one can use the injectivity of $\left.\mathscr{C}_{M \mid X}\right|_{T_{M}^{*}, X^{\prime} \times L} \subset \mathscr{B}_{M \mid X}^{2}$ and $H^{0}\left(\mathscr{B}_{M \mid X}^{a}\right) \subset \mathscr{B}_{M}$ as a substitute of Proposition 1.2. (Note that the latter injectivity follows from the analogous of (1.6) for $\mathscr{B}_{M \mid X}^{2}$ and the concentration in degree 0 for $\mathscr{B}_{M \mid X}^{2}$ (cf. [K-L]). This gives

$$
H^{0}\left(\mathscr{B}_{M \mid X}^{a}\right)_{x}=\left\{u \in\left(\mathscr{B}_{M}\right)_{x}: S S(u) \cap\left(\dot{T}_{M^{\prime}}^{*} X^{\prime} \times L\right)=\varnothing\right\}, \quad x \in M .
$$

REMARK 1.5. For a regular involutive manifold $V$ defined on the whole $\dot{T}_{M}^{*} X$ and satisfying (1.4), we can intrinsically define $\mathscr{B}_{* \mid X}^{a}, *=M, N, \Omega$, by replacing in $(1.5) \bar{*} \times Z$ by $\pi\left(\tilde{V}_{\bar{*}}\right)$ (and $\omega_{L / Z}$ by $\omega_{V / \tilde{F}_{M}}$ ), where $\tilde{V}_{\bar{*}}$ is the union of the leaves of $V^{C}$ issued from $\bar{*} \times_{M} T_{M}^{*} X$; (we also write $\tilde{V}=\tilde{V}_{M}$ ). One can also intrinsically define the right hand sides of (1.11), (1.14) just by replacing $T_{N^{\prime}}^{*} Y^{\prime} \times L$ and $T_{M^{\prime}}^{*} X^{\prime} \times L$ by $\rho \bar{\omega}^{-1}(V)$ and $V$ respectively ( $\rho$ and $\bar{\omega}$ being the natural mappings from $Y \times_{x} T^{*} X$ to $T^{*} Y$ and $T^{*} X$ resp.). It is then clear that if for some coordinates on $M$ we can write

$$
V=\dot{T}_{\mathbb{M}^{\prime}}^{*} X^{\prime} \times L, \quad N=N^{\prime} \times L,
$$

then (1.11) and (1.14) still hold. More generally owing to the invariance of 
$\mathscr{B}_{* \mid M}^{2}$ under contact transformation preserving $V, N \times_{M} V$, and $\omega_{N / M}$ (cf. [U-Z]), one could prove that (1.6) (and the corresponding triangles for $*=M, N$ ) hold. But this refined argument is not needed here.

\section{§2. Extension of solutions with real analytic parameters}

Let $M$ be a real analytic manifold with complexification $X, N$ an analytic hypersurface of $M$ with complexification $Y, \Omega=\Omega^{ \pm}$the two components of $M \backslash N$ at $x, x \in N, \rho$ and $\bar{\omega}$ the canonical mappings from $Y \times_{X} T^{*} X$ to $T^{*} Y$ and $T^{*} X$ respectively.

Let $U \subset M$ be a neighborhood of $x$, and let $V$ be a manifold in $U \times_{M} \dot{T}_{M}^{*} X$. We assume that, in suitable coordinates on $U$ :

$$
\begin{aligned}
& M=M^{\prime} \times L, \quad X=X^{\prime} \times Z, \quad N=N^{\prime} \times L, \\
& V=\dot{T}_{M^{\prime}}^{*} X^{\prime} \times L, \quad \tilde{V}=\dot{T}_{M^{\prime}}^{*} X^{\prime} \times Z, \quad \tilde{V}^{ \pm}=\left(\overline{\Omega^{\prime \pm}} \times{ }_{M^{\prime}} \dot{T}_{M^{\prime}}^{*} X^{\prime}\right) \times Z .
\end{aligned}
$$

Recall the complexes $\mathscr{B}_{M \mid X}^{a}, \mathscr{B}_{\Omega_{\mid X}}^{a}$ (intrinsically associated to $V$ ) and remember (1.11), (1.14). For any $p \in \dot{\pi}^{-1}(x)$ recall the identification $T_{x}^{*} M \hookrightarrow T_{p} T^{*} X$ obtained through the embedding $T^{*} X \times_{X} T^{*} X \hookrightarrow T^{*} T^{*} X$ and the Hamiltonian isomorphism, and observe that $\left(\dot{T}_{N}^{*} M\right)_{x} / R^{+}$is just a pair of vectors $\pm \theta$.

Theorem 2.1. Let $N$ and $V$ be defined, in suitable coordinates by (2.1), and let $\mathscr{M}$ be a coherent $\mathscr{D}_{X}$-module at $x$ which verifies

$$
\begin{gathered}
\pm \theta \notin C_{p}\left(\text { char } \mathscr{M}, \tilde{V}^{\mp}\right) \text { for } \pm \theta \in\left(\dot{T}_{N}^{*} M\right)_{x} / R^{+} \text {and for any } p \in \dot{\pi}^{-1}(x) \cap V, \\
\bar{\omega}^{-1}(\operatorname{char} \mathscr{M}) \cap \rho^{-1} \rho\left(\{x\} \times_{M} V\right) \subset T_{M}^{*} X .
\end{gathered}
$$

Let $S$ be a closed subset of $N$ with $x \in \partial S$ and

$$
i N_{x}^{*}(S) \subset \rho \bar{\omega}^{-1}(V)_{x},
$$

(in the identification $i T^{*} N \simeq T_{N}^{*} Y$ ). We then have, in a neighborhood of $x$,

$$
\operatorname{Hom}_{\mathscr{P}_{X}}\left(\mathscr{M}, \Gamma_{M \backslash S}\left(H^{0}\left(\mathscr{B}_{M \mid X}^{a}\right)\right) \stackrel{\sim}{\sim} \operatorname{Hom}_{\mathscr{P}_{X}}\left(\mathscr{M}, H^{0}\left(\mathscr{B}_{M \mid X}^{a}\right)\right) .\right.
$$

Proof. We shall follow a method closely related to [Kan]. Let $\Omega=\Omega^{ \pm}$ with $\Omega^{+} \cup \Omega^{-}=M \backslash N$ : by reasoning as in $\S 1$, and observing that

$$
\begin{aligned}
R \Gamma_{Q}\left(\mathscr{B}_{M \mid X}^{a}\right) & =R \Gamma_{\left(Q \times_{X} T_{X}^{*} X\right)}\left(\mathscr{B}_{M \mid X}^{2}\right) \\
& =R \Gamma_{\left(\Omega \times_{X} T_{X}^{*} X\right)}\left(\mathscr{B}_{Q_{\mid X}}^{2}\right) \\
& =R \pi_{*} R \Gamma_{\left(T_{X}^{*} X \cup \pi^{-1}(N)\right)}\left(\mathscr{B}_{Q_{\mid X}}^{2}\right),
\end{aligned}
$$

we get a distinguished triangle: 


$$
\mathscr{B}_{\Omega \mid X}^{a} \longrightarrow R \Gamma_{\Omega}\left(\mathscr{B}_{M \mid X}^{a}\right) \longrightarrow R \dot{\pi}_{*} R \Gamma_{\dot{\pi}^{-1(N)}}\left(\mathscr{B}_{\Omega \mid X}^{2}\right) \longrightarrow{ }^{+1} .
$$

Let $\tilde{\Omega}=\Omega^{\prime} \times Z, \tilde{M}=M^{\prime} \times Z, \tilde{N}=N^{\prime} \times Z$, and put $\mathscr{F}=\left.R \operatorname{Hom}_{\mathscr{O}_{X}}\left(\mathscr{M}, \mathscr{C}_{Q_{\mid X}}^{h}\right)\right|_{\tilde{M} \times T^{*} X}$. We note that $(2.2)$ implies $(p ;-\theta) \notin S S(\mathscr{F})$ for $\tilde{\Omega}=\widetilde{\Omega}^{+}$(or $(p ;+\theta) \notin S S(\mathscr{F})$ for $\tilde{\Omega}=\tilde{\Omega}^{-}$). It follows $R \Gamma_{\pi^{-1(\tilde{N})}}(\mathscr{F})=R \Gamma_{\pi^{-1}(\tilde{M} \backslash \bar{\Omega})}(\mathscr{F})=0$. By applying $R \Gamma_{T^{*} X^{\prime} \times L}\left[n_{2}\right]$ to the last equality ( $n_{2}$ being the codimension of $V$ ), we then get, for a neighborhood $U$ of $x$ on $N$,

$$
\left.R \Gamma_{t^{-1}(N)} R \operatorname{Hom}_{\mathscr{I}_{X}}\left(\mathscr{M}, \mathscr{B}_{Q \mid X}^{2}\right)\right|_{U \times \mathbb{K} V}=0 .
$$

Note now that (2.2), (2.3) imply:

$$
\bar{\omega}^{-1}(\operatorname{char} \mathscr{M}) \cap \rho^{-1} \rho\left(U \times_{M} V\right) \subset U \times_{M} V,
$$

which gives, combined with (2.7):

$$
\left.R \dot{\pi}_{*} R \Gamma_{\dot{\dot{x}^{-1}(N)}} R \operatorname{Hom}_{\mathscr{O}_{X}}\left(\mathscr{M}, \mathscr{B}_{Q \mid X}^{2}\right)\right|_{U}=0 .
$$

By (2.6) this implies:

$$
\left.\left.R \operatorname{Hom}_{\mathscr{D}_{X}}\left(\mathscr{M}, \mathscr{B}_{\Omega_{\mid X}}^{a}\right)\right|_{U} \simeq R \operatorname{Hom}_{\mathscr{P}_{X}}\left(\mathscr{M}, R \Gamma_{\Omega}\left(\mathscr{B}_{M \mid X}^{a}\right)\right)\right|_{U} .
$$

Since $\mathscr{B}_{M \mid X}^{a}$ is concentrated in degree $\geqq 0$, then for every $u \in \operatorname{Hom}_{\mathscr{O}_{X}}(\mathscr{M}$, $\left.\Gamma_{M \backslash S}\left(H^{0}\left(\mathscr{B}_{M \mid X}^{a}\right)\right)\right)$, we can define $u^{ \pm}=\left.u\right|_{2^{ \pm}} \in H^{0}\left(R \operatorname{Hom}_{\mathscr{O}_{X}}\left(\mathscr{M}, R \Gamma_{\Omega^{ \pm}}\left(\mathscr{B}_{M \mid X}^{a}\right)\right)\right)$. Owing to (2.9) and (1.11) we get

$$
S S\left(\gamma\left(u^{ \pm}\right)\right) \cap \rho\left(U \times_{M} V\right) \subset T_{Y}^{*} Y .
$$

We also clearly have

$$
\operatorname{supp}\left(\gamma\left(u^{+}\right)-\gamma\left(u^{-}\right)\right) \subset S .
$$

Therefore the conclusion is an immediate consequence of the following two lemmas.

Lemma 2.2 (cf. [Ô]). Let $F$ be a closed set of $M$ and let $u \in\left(\mathscr{B}_{M}\right)_{x}, x \in \partial F$. Then

$$
\left\{\begin{array}{l}
S S(u) \cap N_{x}^{*}(F) \subset\{0\} \\
\operatorname{supp}(u) \subset F
\end{array} \Longleftrightarrow u_{x}=0\right.
$$

Proof. Easy application of Kashiwara-Holmgren's theorem and of the sweeping out procedure by Bony-Schapira.

Lemma 2.3. Let $u \in \operatorname{Hom}\left(\mathscr{M}, \Gamma_{(M \backslash N)}\left(\mathscr{B}_{M}\right)\right)$; then

$$
u \in \operatorname{Hom}_{\mathscr{D}_{X}}\left(\mathscr{M}, H^{0}\left(\mathscr{B}_{M \mid X}^{a}\right)\right) \Longleftrightarrow\left\{\begin{array}{l}
\gamma\left(u^{ \pm}\right) \in H^{0}\left(\mathscr{B}_{N \mid Y}^{a}\right) \\
\gamma\left(u^{+}\right)-\gamma\left(u^{-}\right)=0 .
\end{array}\right.
$$


Proof. It is enough to recall the triangle

$$
\mathscr{C}_{M \mid X} \longrightarrow \mathscr{C}_{\Omega+\mid X} \oplus \mathscr{C}_{\Omega-\mid X} \longrightarrow \mathscr{C}_{N \mid X}[1] \longrightarrow{ }^{+1},
$$

and the estimation

$$
\left.\left.S S(u)\right|_{N} \subset U_{ \pm} S S_{\Omega \pm}^{\mu, 0}\left(u^{ \pm}\right)\right|_{N} \subset \rho^{-1}\left(U_{ \pm} S S\left(\gamma\left(u^{ \pm}\right)\right)\right)
$$

(cf. [S]).

COROLLARY 2.4. In the situation of Theorem 2.1 assume in addition:

$$
\operatorname{Hom}_{\mathscr{D}_{X}}\left(\mathscr{M}, \Gamma_{\tau_{-1}(S)}\left(\mathscr{C}_{M \mid X}\right)\right)_{p}=0 \quad \forall p \in \dot{T}_{M}^{*} X \backslash V, \pi(p)=x .
$$

Then $\left(\right.$ for $\left.\mathscr{A}_{M}=\left.\mathcal{O}_{X}\right|_{M}\right)$

$$
\operatorname{Hom}_{\mathscr{I}_{X}}\left(\mathscr{M}, \Gamma_{(M \backslash S)}\left(\mathscr{A}_{M}\right)\right)_{x} \simeq \operatorname{Hom}_{\mathscr{I}_{X}}\left(\mathscr{M}, \mathscr{A}_{M}\right)_{x} .
$$

By the argument in the proof of (2.7) and by the injectivity of $\left.\mathscr{C}_{M \mid X}\right|_{V} \rightarrow$ $\mathscr{B}_{M \mid X}^{2}$, a sufficient condition for (2.10) is that $\forall p \in\left(\dot{T}_{M}^{*} X \cap \operatorname{ch} \mathscr{M}\right) \backslash V, \pi(p)=x$, there exist a (regular) involutive manifold $V_{p} \subset \dot{T}_{M}^{*} X$ with $V_{p} \ni p$, and invariant under the flow of $N \times_{M} \dot{T}_{M}^{*} X$, and there exist $\theta_{p} \in \dot{T}_{x}^{*} M$ with $S \subset\{m \in M$; $\left.\left\langle m-x, \theta_{p}\right\rangle \geqq 0\right\}$ such that (2.2) is satisfied by $\theta_{p}$ and $\tilde{V}_{p}$.

REMARK 2.5. It is clear from Lemma 2.2 that we can even consider in Theorem 2.1 some singular set $S$ such that $N_{x_{0}}^{*}(S)=T_{x_{0}}^{*} N$ as in the following example:

$$
\begin{aligned}
& M=M^{\prime} \times L \simeq R^{n_{1}} \times R^{n_{2}} \ni x=\left(x^{\prime}, x^{\prime \prime}\right), \quad\left(n_{1} \geqq 2\right), \\
& N=\left\{x_{1}^{\prime}=0\right\}, \quad V=\left\{\eta^{\prime \prime}=0\right\}, \quad S=\{\phi \leqq 0\},
\end{aligned}
$$

where $\phi$ is a $C^{0}$-function on $N$ which verifies

$$
\phi\left(x_{0}\right)=0, \quad \partial_{x_{n_{1}}} \phi\left(x_{0}\right) \neq 0, \quad \partial_{x^{\prime \prime}} \phi\left(x_{0}\right)=0, \quad \partial_{x_{n_{1}}} \phi, \partial_{x^{\prime \prime}} \phi \in C^{0} .
$$

REMARK 2.6. Theorem 2.1 extends the results of [Kan 1], [Ô 1]. These are obtained by choosing $L \simeq R^{n-2}$ and by taking a subset $S \subset N$ with $N^{*}(S)$ $=\left\{d x_{2}\right\}$. Moreover condition (2.2) is replaced there by (classical) microhyperbolicity.

EXAMPLe 2.7. Let $M=M^{\prime} \times L, N=N^{\prime} \times L, M^{\prime}=R \times N^{\prime}, S=S^{\prime} \times L, x_{0}=0$ $\in \partial S$. Take coordinates $\left(x_{1}, x^{\prime}, x^{\prime \prime}\right) \in R \times N^{\prime} \times L$ and $(z, \zeta) \in T^{*} X, z=x+i y$, $\zeta=\xi+i \eta$. Let $V=\left\{\eta^{\prime \prime}=0\right\}$ and consider the $\mathscr{D}_{X}$-module $\mathscr{M}$ associated to the equation

$$
P u=0 \quad \text { for } P=D_{1}^{2}-\left(x_{1}^{r}+x^{\prime s}\right) D^{\prime 2}+D^{\prime 2}, r, s \text { even, } r \geqq 2 .
$$


Then (2.2)-(2.4) hold with $\pm \theta= \pm d x_{1}$ (cf. [S-Z 1]) and thus we get (2.5) and (2.11) (as (2.10) is trivial in the present situation).

EXAmple 2.8. Let $M=M^{\prime} \times L, N=N^{\prime} \times L, M^{\prime}=R^{2} \ni\left(x_{1}, x_{2}\right), N^{\prime}=\{0\} \times R$, $L=R^{n_{2}} \ni x^{\prime \prime}=\left(x_{3}, x_{4}, x^{\prime \prime \prime}\right)$, set $V=\left\{\eta^{\prime \prime}=0\right\}$, and consider the $\mathscr{D}_{X}$-module $\mathscr{M}$ associated to the system

$$
P u=Q u=0 \quad \text { for } P=D_{1}^{3}+D_{3}^{3}+D^{\prime \prime \prime 3}, \quad Q=D_{2}\left(D_{3}^{2}+D_{4}^{2}\right) .
$$

For $S=S^{\prime} \times L$ with $0 \in \partial S$, we have (2.2)-(2.4) and hence also (2.5). Moreover for any $p \notin V$ and for $\pm \theta_{p}= \pm d x_{1}$ or $\pm d x_{2}$ we have (2.2) with $\tilde{V}_{p}=T_{M}^{*} X$. Therefore if we let $S=\left\{x_{1}=x_{2}=0\right\}$, we get (2.10) and (2.11). (This extends Example 1.1 of [Ô 1].)

\section{References}

[Kan 1] A. Kaneko, On continuation of regular solutions of linear partial differential equations, Publ. Res. Inst. Math. Sci., 12 Suppl. (1977), 113-121.

[Kan 2] A. Kaneko, Estimation of singular spectrum of boundary values for some semihyperbolic operators, J. Fac. Sci. Univ. Tokyo, Sect. IA Math., 27 (1980), 401-461.

[K] M. Kashiwara, Talks in Nice, 1972.

[K-L] M. Kashiwara and Y. Laurent, Théorèmes d'annulation et deuxième microlocalisation, Prépubl. d’Orsay, 1983.

[K-S-1] M. Kashiwara and P. Schapira, Microhyperbolic Systems, Acta Math., 142 (1979), 1-55.

[K-S-2] M. Kashiwara and P. Schapira, Microlocal study of sheaves, Astérisque, Soc. Mat. de France, 128 (1985).

[Kat] K. Kataoka, On the theory of Radon transformation of hyperfunctions, J. Fac. Sci. Univ. Tokyo, Sect. IA Math., 28 (1981), 331-413.

[Ô1] T. Ôaku, Removable singularities of solutions of linear partial differential equations-Systems and Fuchsian equations, J. Fac. Sci. Univ. Tokyo Sect. IA Math., 33 (1986), 403-428.

[0̂2] T. Ôaku, F-mild hyperfunctions and Fuchsian partial differential equations, Adv. Studies in Pure Math., 4 (1984), 223-242.

[S] P. Schapira, Front d'onde analytique au bord I and II, C.R. Acad. Sci. Paris, 302 (10)(1986), 383-386, and Sém. E.D.P. Ecole Polyt. Exp. 13, (1986).

[S-Z1] P. Schapira and G. Zampieri, Regularity at the boundary for systems of microdifferential equations, Pitman Res. Notes in Math., 158 (1987), 186-201.

[S-Z2] P. Schapira and G. Zampieri, A new class of N-regular systems, unpublished.

[S-K-K] M. Sato, M. Kashiwara and T. Kawai, Hyperfunctions and pseudodifferential equations, Springer Lecture Notes in Math., 287 (1973), 265-529.

[U-Z] M. Uchida and G. Zampieri, 2nd microfunctions at the boundary, Res. Inst. Math. Sci., $26 n^{\circ} 2$ (1990), 205-219. 